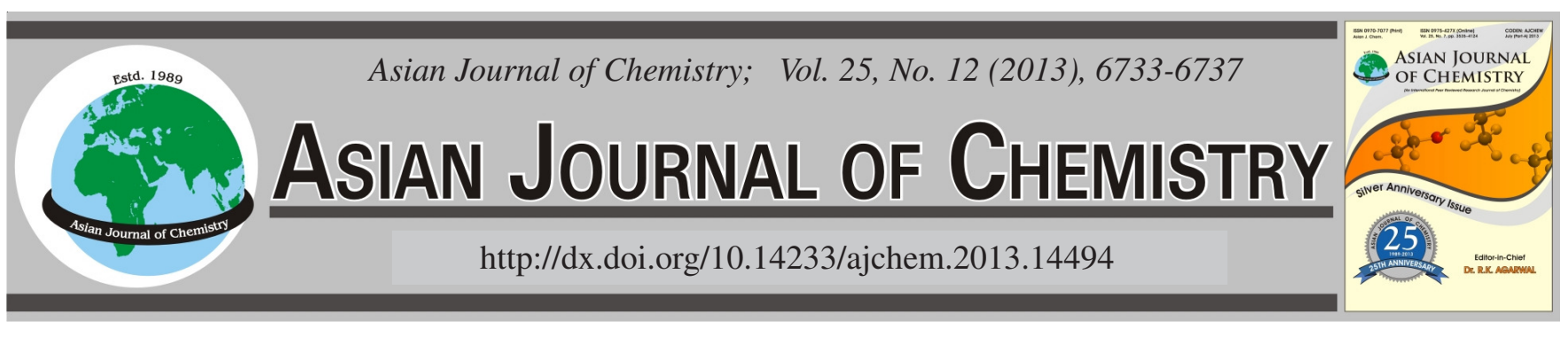

\title{
Acclimation of Anaerobic Culture Degrading 2,4-Dichlorophenol and Its Biodegradation
}

\author{
T. CHENG ${ }^{1, *}$, C. CHEN ${ }^{2}$ and T. PAN ${ }^{1}$
}

${ }^{1}$ Department of City Science, Jiangsu City Vocational College, Nanjing 210036, P.R. China

${ }^{2}$ School of Biology and Chemical Engineering, Jiangsu University of Science and Technology, Zhenjiang 212018, P.R. China

*Corresponding author: Fax: +86 25 86496516; Tel: +86 25 86496580; E-mail: wnchengting@yahoo.com.cn

(Received: 28 August 2012;

Accepted: 29 May 2013)

AJC-13560

\begin{abstract}
The experiment was conducted to examine the biodegradation of 2,4-dichlorophenol (2,4-DCP) and the variation of COD concentration during the acclimation period of anaerobic microorganism. The effect of acclimation substrates and acclimated culture on the 2,4-DCP degradation was also investigated. The results showed that, the anaerobic microorganism had the potential of degrading chlorophenols. During the acclimation period, the anaerobic culture adapted to the toxicity of 2,4-DCP gradually. The degradation capability and degradation rate of 2,4-DCP improved with the extension of acclimation time. The 2,4,6-trichlorophenol (2,4,6-TCP) and 2,4-DCP acclimated culture for the 2,4-DCP degradation was superior to the 2,4-DCP acclimated culture during the acclimation period. The COD removal rate was enhanced with the long-term coexistence of 2,4,6-TCP and 2,4-DCP. Moreover, Compared to the fresh culture, the acclimated culture improved the 2,4-DCP degradation obviously and the optimal acclimated microorganism was the culture acclimated to 2,4,6-TCP and 2,4-DCP. The COD removal rate in different systems had a relation to the 2,4-DCP degradation in some extent.
\end{abstract}

Key Words: Chlorophenols, 2,4-Dichlorophenol, 2,4,6-Trichlorophenol, Acclimation sludge, Biodegradation.

\section{INTRODUCTION}

Chlorophenols (CPs) are exenobiotic pollutants that enter the environment through various industrial operations such as the production of resins, nylon, plastics, antioxidants, lubricant additives, wood protectors, bleached pulp, pesticides, textile, dyes, explosives, disinfectants or biocides, etc. ${ }^{1,2}$. Chlorophenols are generally recognized for their toxicity and recalcitrant nature. Some of the characteristics of chlorophenols, such as 2,4-DCP, 2,4,6-TCP, pentachlorophenol (PCP) are their acute toxicity and poor biodegradability ${ }^{3-5}$. Five chlorophenols are listed by the U.S. Environmental Protection Agency (US EPA) as priority pollutants. Due to their high toxicity, persistence and bioaccumulation in aquatic organisms, nowadays, their disposal has become a major environmental concern and there is a need for efficient methods to eliminate poorly biodegradable chlorophenols from industrial effluents and polluted groundwater. Degradation of these important problematic environmental contaminants has been studied extensively by a wide variety of methods, including biological treatment, conventional physical and chemical techniques, chlorination and adsorption ${ }^{3,6,7}$. However, robust and cost effective treatment of the pollutants has still to be implemented.

To date, anaerobic biological process is one of the preferred approaches in removing chlorophenols from the wastewaters.
Microorganisms have the advantage that they use chlorophenols as carbon source, which leads to a high degree of elimination. Dai et al. ${ }^{8}$ suggested that chlorophenols was extremely resistant to oxidative degradation, whereas it become easier in anaerobic conditions. Said et al. ${ }^{9}$ advised that anaerobic biodegradation and biotransformation could be considered as a promising means for bioremediation treatments of persistently polluted environments in subsurface soil and sediments. Anaerobic biodegradation of chlorophenols is initiated by a process known as reductive dechlorination, in which the chlorine substituent is removed with concurrent addition of electrons to the molecule. Under anaerobic conditions, bacteria can dechlorinate various chlorophenols to produce less-chlorinated phenol $^{10}$. Nicholson et al. ${ }^{11}$ suggested that the anaerobic digester sludge fed 5,300 mg of acetate per liter, 3.4 $\mu \mathrm{M}$ pentachlorophenol and nutrients for 10 days transformed pentachlorophenol by sequential ortho dechlorinations to produce 2,3,4,5-tetrachlorophenol and 3,4,5-trichlorophenol. The investigations indicated that the inoculation microbe, domestication substances and domestication period could impact the degradability, degradation rate, degradation pathway of chlorophenols ${ }^{12,13}$. Boyd and Shelton ${ }^{12}$ investigated the anaerobic biodegradation of monochlorophenol and dichlorophenol isomers by unacclimated and acclimated sludge, the results demonstrated the sludge acclimated to 
4-chlorophenol (4-CP) could degrade 2-chlorophenol (2-CP), 3-chlorophenol (3-CP), 4-chlorophenol, 2,4-DCP and 3,4DCP, the sludge acclimated to 2-chlorophenol cross-acclimated to 4-chlorophenol could degrade 2,4-DCP, but did not utilize 3 -chlorophenol. In recent years, the researches related on the degradation of chlorophenols are mostly focused on the degradation activities of acclimation sludge. However, the researches about the characteristic of acclimation process were relatively insufficient. Usually, long acclimation period was needed in order for the anaerobic sludge to function properly and to achieve chlorophenols degradation.

In this work, therefore, we focused on the biodegradation of 2,4-DCP during the acclimation period of anaerobic sludge. In addition, to examine the effect of acclimation substrates on the degradation of target contaminant $(2,4-\mathrm{DCP})$, the sludge was acclimated to individual 2,4-DCP, 2,4,6-TCP and 2,4-DCP, respectively. The impact of acclimated culture on the 2,4-DCP degradation was also investigated in the test.

\section{EXPERIMENTAL}

Microorganisms: A mixed anaerobic sludge used for this research was developed from a full-scale internal circulation reactor treating dye wastewater. The anaerobic sludge was first fed with 2,500 mg of glucose per liter as the carbon source for two weeks, which enhanced the biological activity and the chemical oxygen demand (COD) removal rate was over $80 \%$ before the experiments.

Acclimation of anaerobic sludge: The acclimation of mixed culture was inoculated with the anaerobic sludge in 2,500 L flask. The sludge was acclimated to 2,4-DCP, 2,4,6TCP and 2,4-DCP, respectively. Each flask was fed glucose, chlorophenols stock solution and nutrients. $\mathrm{NaHCO}_{3}$ was added to maintain a buffering capacity. Then the liquid was purged with nitrogen for $10 \mathrm{~min}$ to remove any residual dissolved oxygen completely,flasks were sealed with rubber stoppers and incubated at $37^{\circ} \mathrm{C}$ for more than 2 months. During the acclimation period, the concentration of 2,4-DCP and 2,4,6TCP was increased gradually, nutrient and buffer solution was added to supply the nutrition for the microorganism. Meanwhile, a regular sample was taken from each flask for 2,4DCP and COD detection. The nutrient medium in flasks contained (mg L $\left.{ }^{-1}\right): \mathrm{KH}_{2} \mathrm{PO}_{4} 54, \mathrm{~K}_{2} \mathrm{HPO}_{4} 70, \mathrm{NH}_{4} \mathrm{Cl} 106$, $\mathrm{CaCl}_{2} \cdot 2 \mathrm{H}_{2} \mathrm{O} 15, \mathrm{MgCl}_{2} \cdot 6 \mathrm{H}_{2} \mathrm{O} 20$. The trace element solution contained (mg L $\left.{ }^{-1}\right): \mathrm{CoCl}_{2} \cdot 6 \mathrm{H}_{2} \mathrm{O} 500, \mathrm{NiCl}_{2} \cdot 6 \mathrm{H}_{2} \mathrm{O} 50, \mathrm{Na}_{2} \mathrm{SeO}_{3}$ $50, \mathrm{CuCl}_{2} \cdot 2 \mathrm{H}_{2} \mathrm{O} 30, \mathrm{ZnCl}_{2} 50, \mathrm{H}_{3} \mathrm{BO}_{3} 50, \mathrm{MnCl}_{2} \cdot 4 \mathrm{H}_{2} \mathrm{O} 500$, $\left(\mathrm{NH}_{4}\right)_{6} \mathrm{Mo}_{7} \mathrm{O}_{24} \cdot 2 \mathrm{H}_{2} \mathrm{O} 10$. Additionally, the concentration of $\mathrm{NaHCO}_{3}$ buffer solution was $1,000 \mathrm{mg}$ per liter and the biomass concentration in each flask was $c a$. 4,200 mg per liter based on the volatile suspended solids (VSS) contents of anaerobic sludge.

Biodegradation of 2,4-DCP by fresh and acclimated culture: Batch experiments were conducted using $250 \mathrm{~mL}$ serum bottles at $37^{\circ} \mathrm{C}$. The mixed culture, which acclimated to 2,4-DCP, 2,4,6-TCP and 2,4-DCP, respectively, was transferred to serum bottle with $50 \mathrm{~mL}$ nutrient medium and $2 \mathrm{~mL}$ trace element solution. Each bottle was spiked with 2,4-chlorophenol stock solution to give an initial concentration of 30 $\mathrm{mg} / \mathrm{L}$. $\mathrm{NaHCO}_{3}(1,000 \mathrm{mg} / \mathrm{L})$ was also added to maintain a buffering capacity. Bottles were then filled with deionized water and $\mathrm{pH}$ was adjusted with $\mathrm{HCl}$ and $\mathrm{NaOH}$ solution. The Initial $\mathrm{pH}$ was adjusted to 7 in all batch experiments. The liquid was purged with nitrogen for $10 \mathrm{~min}$ to remove any residual dissolved oxygen completely,and then bottles were sealed with rubber stoppers and placed on a platform shaker and shaken continuously at $120 \mathrm{rpm}$ over the course of experiments. The biomass concentration in each bottle was approximately $360 \mathrm{mg} / \mathrm{L}$ based on the volatile suspended solids (VSS) contents of anaerobic sludge. A regular sample was taken from each bottle for 2,4-DCP and COD detection.

Analytical methods: The sample was taken from flask or bottle using a glass syringe. Analysis for 2,4-DCP was performed using a Agilent LC-1260 HPLC system, equipped with a Lichrospher $\mathrm{C}_{18}$ inverse phase column. An L-2400 UV detector was used for the analysis and the detection wavelength was $280 \mathrm{~nm}$. The HPLC mobile phase was the mixture of purified water $(20 \%)$ and methanol $(80 \%)$ at flow rate of $0.8 \mathrm{~mL} \mathrm{~min}^{-1}$. The injection volume was $5 \mu \mathrm{L}$ with an autosampler. Prior to HPLC analysis, sample solutions were filtered by $0.45 \mu \mathrm{m}$ membrane. Standard potassium dichromate method was used for the determination of chemical oxygen demand. The biomass was measured as volatile suspended solids using standard methods.

\section{RESULTS AND DISCUSSION}

Biodegradation of pollutant during the acclimation period: The test was to investigate the biodegradation of 2,4DCP by the microorganism acclimated to 2,4-DCP, 2,4,6-TCP and 2,4-DCP, respectively. Fig. 1 showed the 2,4-DCP degradation during the acclimation period. Fig. 1 indicated that the anaerobic microorganism acclimated to chlorophenols began degrading 2,4-DCP almost without lag phase. The anaerobic microorganism demanded for a long time (more than 2 months) to attain a higher efficiency to degrade target contaminant. With the increasing of chlorophenols concentration in the process of acclimation, the anaerobic sludge adapted to the toxicity of chlorophenols gradually and the biodegradation ability for target pollutant was also improved. This suggested that the anaerobic microorganism had the potential of degrading chlorophenols and inducing the potential of degradation demanded a relatively short time. However, it demanded a long time to enrich a certain amount of the microorganism having the degradation activity.

Additionally, the degradation effect of the culture acclimated to 2,4-DCP was superior to the culture acclimated to 2,4,6-TCP and 2,4-DCP in the early acclimation period (1-20 days). While the acclimation period was prolonged more than 30 days, the degradation ability of 2,4,6-TCP and 2,4-DCP acclimated culture improved obviously and the degradation effect was better than the culture acclimated to individual 2,4DCP in the acclimation period of 31-70 days. Adding $26 \mathrm{mg}$ 2,4-DCP per liter again in the acclimation period of 60 days, the degradation rate of target contaminant reached more than $90 \%$ in the 2,4,6-TCP and 2,4-DCP acclimated culture. While the degradation rate was only $68 \%$ in the $2,4-\mathrm{DCP}$ acclimated culture. That could be demonstrated in the early acclimation period the degradation of target contaminant was impacted by 


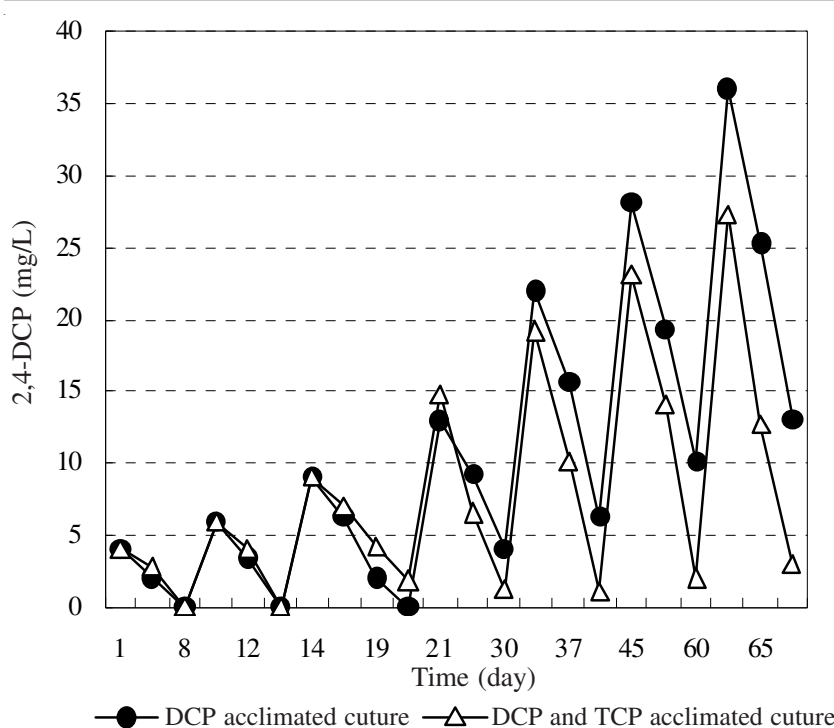

Fig. 1. 2,4-DCP degradation during the acclimation period

the accumulation of 2,4,6-TCP and 2,4-DCP toxicity. However, the mixed acclimated microorganism adapted to the toxicity of chlorophenols gradually with the extension of acclimation time. The co-existence of 2,4,6-TCP and 2,4-DCP accelerated the degradation of 2,4-DCP. Fen-xia and Dongsheng ${ }^{14}$ pointed out that the acclimation of individual chlorophenol in general demanded a lag phase to begin to degrade pollutants and a lag phase was about 2 weeks. Furthermore, the concentration of chlorophenols was low (less than $10 \mathrm{mg}$ / L) in the early acclimation period of 20 days and the rapid decreasing of 2,4-DCP perhaps due to some adsorption of pollutant.

Fig. 2 showed the degradation rate of 2,4-DCP during the acclimation period. Fig. 2 suggested that the degradation rate of target pollutant showed a rising trend with the extension of acclimation time. The degradation rate of 2,4-DCP by the culture acclimated to 2,4-DCP improved obviously. The degradation rate improved from $0.57 \mathrm{mg} \mathrm{L}^{-1} \mathrm{~d}^{-1}$ (1-8 days) to $1.27 \mathrm{mg} \mathrm{L}^{-1} \mathrm{~d}^{-1}$ (45-60 days) and to $2.39 \mathrm{mg} \mathrm{L}^{-1} \mathrm{~d}^{-1}$ (60-70 days). Moreover, the degradation rate of $2,4-\mathrm{DCP}$ by the culture acclimated to 2,4,6-TCP and 2,4-DCP was higher than the culture acclimated to $2,4-\mathrm{DCP}$. The degradation rate improved from $0.57 \mathrm{mg} \mathrm{L}^{-1} \mathrm{~d}^{-1}$ (1-8 days) to $1.47 \mathrm{mg} \mathrm{L}^{-1} \mathrm{~d}^{-1}$ (45-60 days) and to $2.64 \mathrm{mg} \mathrm{L}^{-1} \mathrm{~d}^{-1}$ (60-70 days).

That indicated the culture acclimated to $2,4,6-\mathrm{TCP}$ and 2,4-DCP presented higher potentiality for the degradation of 2,4-DCP. Quan et al. ${ }^{15}$ suggested that, with the long-term coexistence of 4-chlorophenol and 2,4-DCP, the degradation rate of 2,4-DCP was higher than the situation of individual 2,4-DCP as acclimation substrate. The molecular structure of 2,4,6-TCP was similar to 2,4-DCP, that inferred the long-term coexistence of 2,4,6-TCP and 2,4-DCP induced more enzyme or enzyme system for the anaerobic microorganism degrading 2,4-DCP and that promoted the biodegradation of target contaminant.

Variation of COD concentration during the acclimation period: Fig. 3 showed the variation of COD concentration during the acclimation period. Fig. 3 suggested that the variation of COD concentration was impacted by the addition of

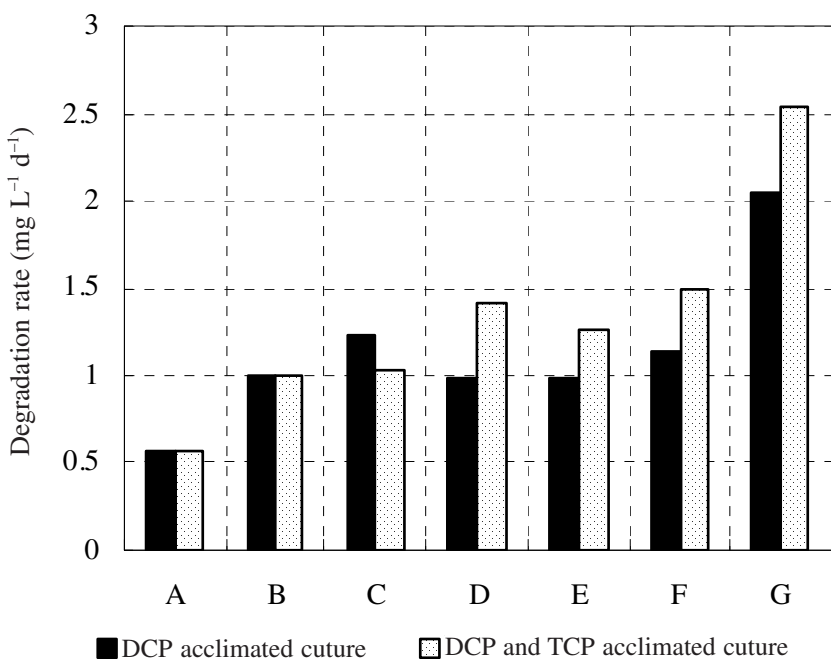

Fig. 2. Degradation rate of 2,4-DCP during the acclimation period

chlorophenols. Combined the variation of 2,4-DCP concentration during the acclimation period (Fig. 1), that inferred the degradation rate of COD decreased obviously when the 2,4DCP concentration was more than $6 \mathrm{mg} / \mathrm{L}$. It indicated the addition of chlorophenols caused the inhibition of microorganism degrading glucose. While with the extension of acclimation time, the anaerobic culture adapted to the toxicity of chlorophenols gradually and the degradation rate of COD also recovered. Additionally, in the early 16 days of acclimation period, the COD degradation rate in the anaerobic microorganism system of 2,4,6-TCP and 2,4-DCP acclimated culture was slower than the system of 2,4-DCP acclimated culture. However, the COD degradation rate in the system of 2,4,6-TCP and 2,4-DCP acclimated culture was higher than the system of 2,4-DCP acclimated culture with the extension of acclimation time (28-65 days). That suggested the anaerobic microbial community adapted to the toxicity gradually with the long-term coexistence of 2,4,6-TCP and 2,4-DCP and the degradation effect of target contaminant and organic substrates was better. Combined the experimental results of Fig. 3 with Fig. 1, indicated the variation of COD concentration had a relation to the biodegradation of 2,4-DCP in some extent in the same reaction system. This observation coincided with the results of Li et al. ${ }^{16}$ that acclimated to pentachlorophenol with glucose as co-metabolism substrate. It inferred the metabolic enzyme of anaerobic microorganism was not absolute specificity. With the degradation of supplementary carbon source by the metabolic enzyme, target contaminant (2,4-DCP) was also degraded. Moreover, because of the COD concentration was relatively higher, the anaerobic sludge system maintained above $1,000 \mathrm{mg} / \mathrm{L}$ COD concentration.

Effect of different culture on the biodegradation of 2,4DCP: Fig. 4 showed the effect of different culture on the degradation of 2,4-DCP. In the test, the initial $\mathrm{pH}$ of all systems was 7 and the inoculation amount of anaerobic culture was 276 mg VSS per liter. Fig. 4 suggested the acclimated culture improved the degradation effect of 2,4-DCP obviously compared to the fresh culture. The degradation effect of the culture acclimated to 2,4-DCP and 2,4,6-TCP was the best than the other cultures. When the 2,4-DCP and 2,4,6-TCP acclimated culture degraded 2,4-DCP in $190 \mathrm{~h}$, the degradation 


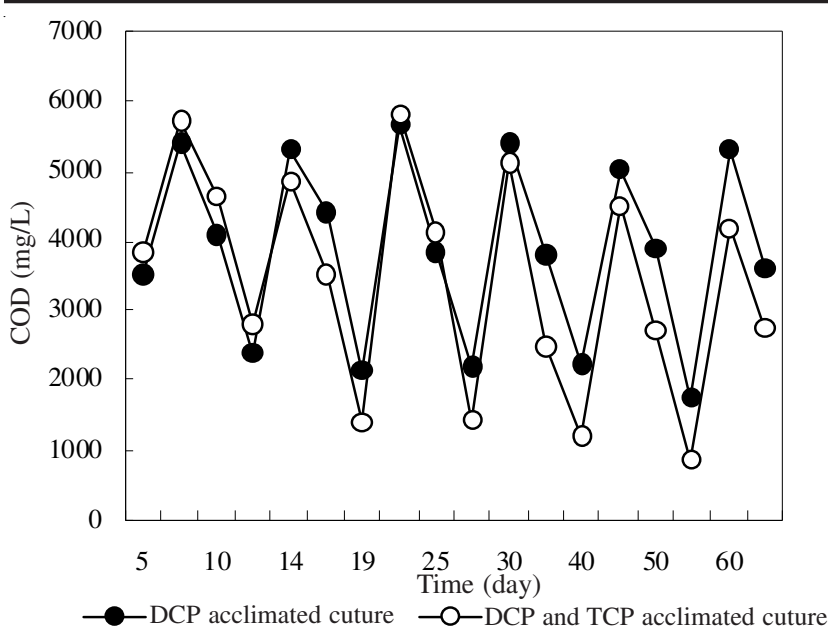

Fig. 3. Variation of COD concentration during the acclimation period

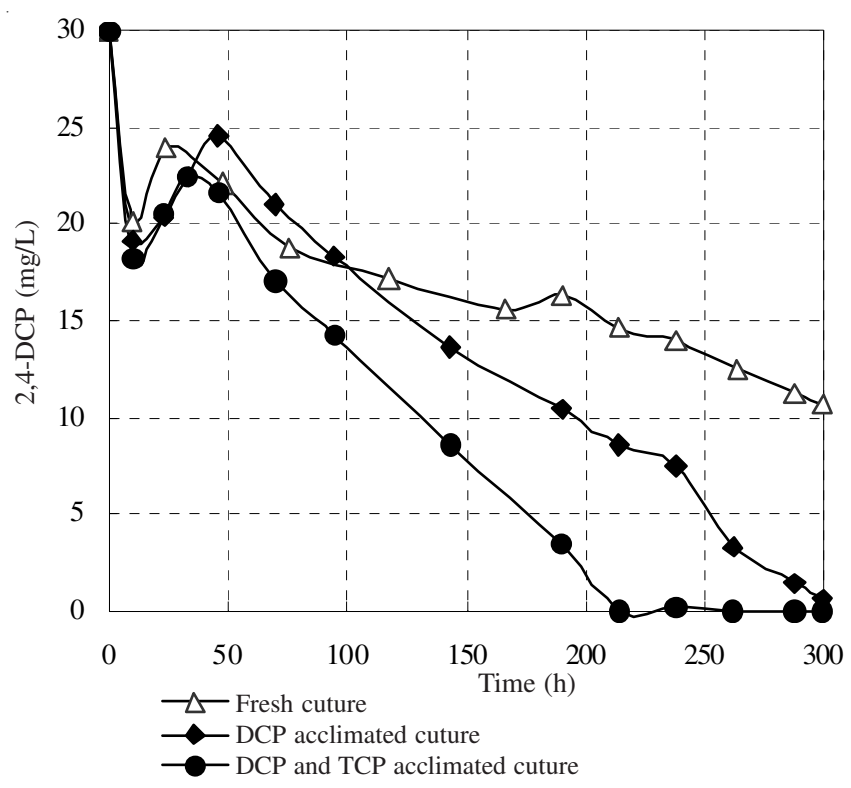

Fig. 4. Effect of different culture on the biodegradation of 2,4-DCP

rate of target contaminant was more than $90 \%$. The 2,4-DCP was completely degraded in $214 \mathrm{~h}$. In contrast, the degradation rate of 2,4-DCP was $65 \%$ in $190 \mathrm{~h}$ by the $2,4-\mathrm{DCP}$ acclimated culture and it reached $90 \%$ in $288 \mathrm{~h}$. The 2,4-DCP was degraded completely In $300 \mathrm{~h}$. However, in the system of fresh (unacclimated) culture, the degradation rate of 2,4-DCP was very low. The 2,4-DCP degradation rate was only $45 \%$ in 190 $\mathrm{h}$ and the residual contaminant was still $30 \%$ in $300 \mathrm{~h}$. That perhaps different acclimation and cultivation made the different microbial community and thus the biodegrability of microbial community for the chlorophenols was distinct.

Moreover, the anaerobic degradation of 2,4-DCP by the three cultures was experienced the process of adsorption, desorption and biodegradation. In the early reaction period, the anaerobic microorganism mainly adsorbed the target contaminant. The system reached equilibrium in a short time (23-33 h) and began desorbing the pollutant. Then in a relatively long time the microorganism accomplished the degradation of pollutant. Fig. 4 also showed that the anaerobic microorganism was experienced a long time lag phase to degrade 2,4-DCP. That inferred the toxicity of 2,4-DCP with the concentration of $30 \mathrm{mg} / \mathrm{L}$ was relatively higher to the microorganism. The microorganism demanded a long adaptation period to begin to degrade pollutant.

To reflect the anaerobic biodegradation of organics, the variation of COD concentration in different reaction systems was measured at the same time. Fig. 5 showed the removal rate of COD in different anaerobic systems. Fig. 5 showed that the removal rate of COD in different degradation systems had a relation to the biodegradation of 2,4-DCP in some extent. The degradation of organics was very low in the system of fresh culture and the removal rate of COD was only $51 \%$. The removal rate of COD was $71 \%$ in the system of 2,4-DCP acclimated culture. In addition, the degradation of organics was the highest in the system of 2,4-DCP and 2,4,6TCP acclimated culture, the removal rate of COD was more than $80 \%$.

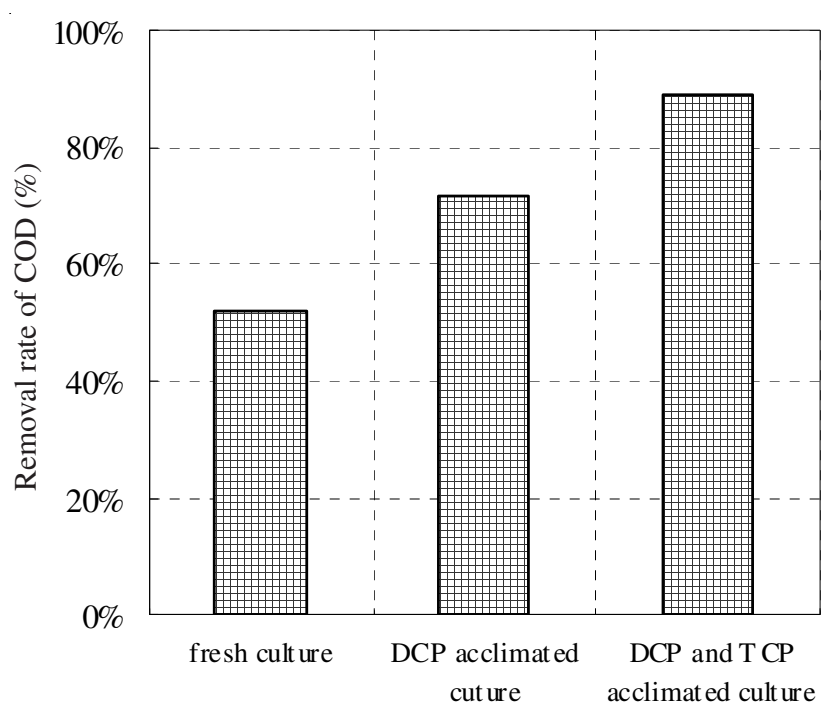

Fig. 5. Removal rate of COD in different anaerobic systems

\section{Conclusion}

Anaerobic microorganism had the potential of degrading chlorophenols. Inducing the potentiality of microorganism demanded a relatively short time, but it demanded a long time to enrich a certain amount of the microbial community having the degradation activity. The degradation ability of anaerobic culture for the 2,4-DCP improved gradually during the acclimation period and the 2,4-DCP degradation rate was also increased. In addition, the long-term coexistence of 2,4,6-TCP and 2,4-DCP induced more enzyme or enzyme system for the microorganism degrading 2,4-DCP and the degradation effect of 2,4,6-TCP and 2,4-DCP acclimated culture was better than the culture acclimated to individual 2,4-DCP during the acclimation period in general. The addition of chlorophenols caused the inhibition of microorganism degrading glucose in the early acclimation process. However, with the extension of acclimation time, the anaerobic culture adapted to the toxicity of chlorophenols gradually and the degradation rate of COD also recovered. Compared to the fresh culture, the acclimated culture improved the degradation effect of 2,4-DCP obviously. The degradation effect of the culture acclimated to 2,4-DCP and 2,4,6-TCP was superior to the other cultures. Moreover, 
the degradation of organics was the highest in the system of 2,4-DCP and 2,4,6-TCP acclimated culture, the removal rate of COD was more than $80 \%$. The removal rate of COD in different degradation systems had a relation to the biodegradation of 2,4-DCP in some extent.

\section{ACKNOWLEDGEMENTS}

The authors gratefully acknowledged the financial support of the "Twelfth Five-Year Plan" Key Task of Jiangsu City Vocational College (Grant No. 12SEW-Z-004).

\section{REFERENCES}

1. R. Valo, V. Kitunen and M.S. Salkinoja-Salonen and S. Raisanen, Water Sci. Technol., 17, 1384 (1985).

2. M. Czaplicka, Sci. Total Environ., 322, 21 (2004).

3. X. Zhang and J. Wiegel, Appl. Environ. Microbiol., 56, 1119 (1990).

4. A. Breitenstein, A. Saano and M. Salkinoja-Salonen, J.R. Andreesen and U. Lechner, Arch. Microbiol., 175, 133 (2001).

5. P. Juteau, R. Beaudet, G. McSween, F. Lépine, S. Milot and J.-G. Bisaillon, Appl. Microbiol. Biotechnol., 44, 218 (1995).

6. W. Chu and C.K. Law, Water Res., 37, 2339 (2003).

7. N. Wang, X.Y. Li, Y.X. Wang, X. Quan and G.H. Chen, Chem. Eng. J., 146, 30 (2009).

8. Y.Z. Dai, H.C. Shi and J.P. Ji, Environ. Sci., 21, 40 (2000) (in Chinese).

9. S. El-Fantroussi, H. Naveau and S.N. Agathos, Biotechnol. Progr., 14, 167 (1998).

10. C.C. Chang, S.K. Tseng, C.C. Chang and C.-M. Ho, Bioresour. Technol., 90, 323 (2003).

11. D.K. Nicholson, S.L. Woods, J.D. Istok and D.C. Peek, Appl. Environ. Microbiol., 58, 2280 (1992).

12. S.A. Boyd and D.R. Shelton, Appl. Environ. Microbiol., 47, 272 (1984).

13. D.S. Shen, X.Y. Xu, X.S. Feng, Acta Scient. Circumst., 16, 299 (1996) (in Chinese).

14. F.-X. Ye and D.-S. Shen, Chemophere, 54, 1573 (2004).

15. X.C. Quan, H.C. Shi, J.L. Wang and Y. Qian, Acta Scient. Circumst., 23, 69 (2003) (in Chinese).

16. P. Li and J.X. Liu, China Water Wastewater, 20, 35 (2004) (in Chinese). 